\title{
Estimate of Global and Regional Distributions of Ionospheric Electrodynamic Quantities from EISCAT Radar and Ground Magnetometer Data
}

\author{
Mariko SATO and Y. KAMIDE \\ Solar-Terrestrial Environment Laboratory, Nagoya University, 3-13 Honohara, Toyokawa, Aichi 442, Japan
}

(Received February 28, 1995; Revised May 23, 1995; Accepted May 30, 1995)

\begin{abstract}
A new scheme is presented to estimate both global and localized distributions of ionospheric electric fields and currents by connecting two advanced magnetogram-inversion methods. It is demonstrated that a suitable combination of data from ground-based magnetometers and those from the EISCAT radar and polar orbiting satellites is able to provide us with valuable information on the electrodynamic properties in a localized area associated with substorm intensifications as well as on the global patterns of ionospheric convection. Along with the concept and the practical procedure of the new scheme, its capability in studying the substorm is addressed.
\end{abstract}

\section{Introduction}

Determining the variable electromagnetic state of the polar ionosphere is essential in the dynamics of magnetosphere-ionosphere coupling. In fact, since constantly changing geomagnetic fields are being monitored routinely by a large number of ground-based magnetometers, magnetic field data are widely used in understanding variations of the global distribution of ionospheric properties, for example, of plasma convection patterns. It should be noted, however, that the ground magnetometers do not directly measure ionospheric currents; there is no unique way to determine the distribution of field-aligned and ionospheric currents only from magnetic field observations made on the earth's surface.

On the other hand, the incoherent scatter radar is able to provide relatively direct measurements of ionospheric quantities in spite of the fact that the spatial coverage by one radar is rather limited. It is thus very useful that we combine ground magnetometer data, giving global but indirect information, with radar data for small-scale but relatively direct information on the ionosphere, and also with in situ measurements from satellites.

Baumjohann et al. (1981) used two-dimensional distributed data of the magnetic and electric fields from the Scandinavian Magnetometer Array and the STARE radar, respectively, to obtain the threedimensional current system associated with active auroral forms. They emphasized the importance of studies on small-scale electrodynamics of the auroral ionosphere for a better understanding of the physics of substorm processes, showing that highly localized and intense upward field-aligned currents flow at the western edge of active regions. Another important and unsettled subject in substorm studies is the relationship between such localized manifestations of substorms and the large-scale plasma convection.

We attempt in this paper to derive simultaneously global and localized patterns of the electric fields and currents by combining two magnetogram-inversion methods, i.e., KRM (Kamide, Richmond and Matsushita; Kamide et al., 1981) and AMIE (Assimilative Mapping of Ionospheric Electrodynamics; Richmond and Kamide, 1988) techniques. Most recently, Sato et al. (1995) have applied this new scheme to one case to discuss the relative importance of electric fields and conductances in generating the auroral electrojets.

The present paper aims at reviewing the concept and technical details of the scheme, discussing its advantages and limitations as well. This scheme is applied to a substorm event, which occurred in northern 
Scandinavia near the EISCAT radar. Along with the results of our application, we also show how crucial it is to include data from radar observations in this new powerful scheme.

\section{New Scheme}

In substorm research it is important to identify a particular substorm-intensified region within the framework of global convection patterns. Our new scheme is quite useful for this purpose. Figure 1 shows one such example, in which we present (a) the electric potential distribution over the entire polar region, and (b) the distribution in a localized region. Owing to the highly non-uniform distribution of magnetometers, "global" calculations tend to smear out small-scale structures of ionospheric quantities even in the region where magnetic observatories are densely distributed.

A regional estimation is applied to the localized region where data from numerous magnetometer stations and from the EISCAT radar are available. In (b), some small-scale structures, such as deformed and additional contours, are generated, a feature which cannot be seen in (a). For the regional estimation shown in (b), (1) the boundary conditions for the current function and the electric potential are taken from the global distribution calculated with the AMIE, and (2) small-scale conductance distributions are employed. We describe in the following the essence of magnetogram-inversion techniques and how (1) and (2) are dealt with in our scheme.

\subsection{Basic concept}

The basic equations for the ionospheric electrodynamic quantities have been given, for example, by Kamide and Baumjohann (1993), and Untiedt and Baumjohann (1993). The ionospheric current $\boldsymbol{J}$ can be separated into a divergence-free part $\boldsymbol{J}_{\mathrm{T}}$ and a curl-free part $\boldsymbol{J}_{\mathrm{P}}$ :

$$
\boldsymbol{J}=\boldsymbol{J}_{\mathrm{T}}+\boldsymbol{J}_{\mathrm{P}}
$$

The second term $\boldsymbol{J}_{\mathrm{P}}$ is called the "potential" current and can be considered as a current closing with the

\section{ELECTRIC POTENTIAL}

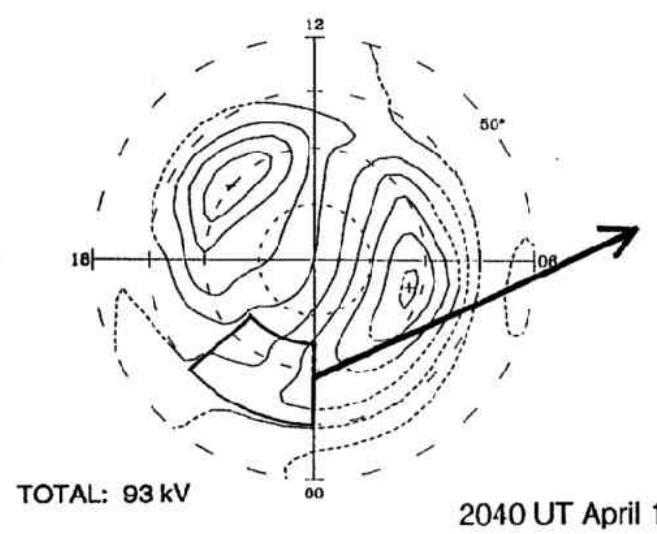

(a)

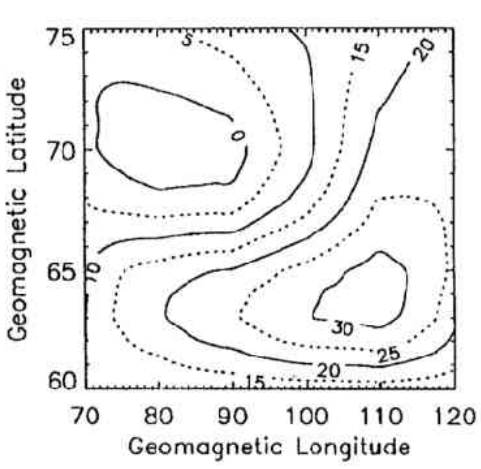

(b)

Fig. 1. Examples of the results from the new scheme for obtaining global and localized distributions of the electrodynamic quantities in the high-latitude ionosphere. This scheme combines the AMIE and KRM algorithms, as described in this paper: (a) Global electric potential pattern (10 kV contour interval) obtained by the AMIE technique. (b) Electric potential distribution ( $5 \mathrm{kV}$ contour interval) derived by the KRM method for a localized region. In the localized distribution (b), smallscale structures such as a deformation of the potential contour lines are seen, which are not evident in (a). 
field-aligned currents. $\boldsymbol{J}_{\mathrm{T}}$ is the so-called equivalent current whose associated magnetic field matches the external portion of the observed magnetic field, and can be expressed in terms of the current function $\psi$ :

$$
\boldsymbol{J}_{\mathrm{T}}=n_{r} \times \nabla \psi
$$

where $n_{r}$ is the unit vector in the radial direction. We assume that the earth's magnetic field lines are effectively perpendicular to the ionospheric plane.

The "total" ionospheric current is related to the electrostatic potential $\phi$ as:

$$
\boldsymbol{J}=-\left(\Sigma_{\mathrm{P}} \nabla \phi+\Sigma_{\mathrm{H}}\left(\nabla \phi \times n_{r}\right)\right)
$$

where $\Sigma_{\mathrm{P}}$ and $\Sigma_{\mathrm{H}}$ are the height-integrated Pedersen and Hall conductivities, respectively.

By taking the curl of (1) and (3) and equating their right-hand sides, the following second-order differential equation can be obtained for the electric potential:

$$
\Sigma_{\mathrm{H}}\left(\nabla^{2} \phi\right)+\left(\nabla \Sigma_{\mathrm{H}}\right) \cdot(\nabla \phi)-n_{r} \cdot\left(\left(\nabla \Sigma_{\mathrm{P}}\right) \times \nabla \phi\right)=-\nabla^{2} \psi
$$

Although the basic concepts of the KRM and AMIE are similar, their practical procedures are different. In the KRM algorithm, Eq. (4) is solved numerically for given inputs of the current function $\psi$ estimated from ground magnetograms. In this process, the Hall and Pedersen conductances must be given.

On the other hand, in the AMIE algorithm, mutually consistent patterns of the electric potential, the ionospheric current, the field-aligned current and magnetic perturbations on the ground and at the satellite heights are determined by a statistical method of optimization. For the AMIE calculation, many different types of simultaneously measured parameters can be used, such as electric fields and conductances from radar and satellites, field-aligned currents from satellites and ionospheric currents from radar in addition to ground-based magnetic perturbations. Our practical procedure is summarized in Fig. 2, where it is

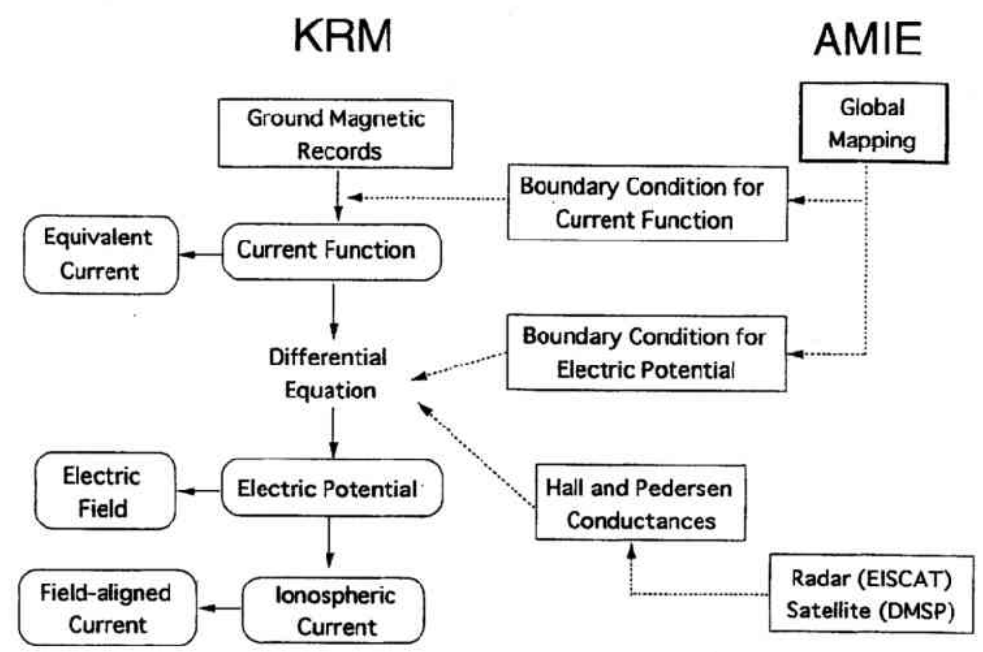

Fig. 2. A flow chart showing the procedure of the new scheme. The KRM algorithm is used for the regional estimation which is applied to northern Scandinavia. Input parameters for KRM are the Hall and Pedersen conductances and the current function determined from ground magnetometer data in the localized region. The AMIE technique derives the global distribution of the electrodynamic quantities, giving the boundary conditions in the current function and the electric potential for the regional estimation. 


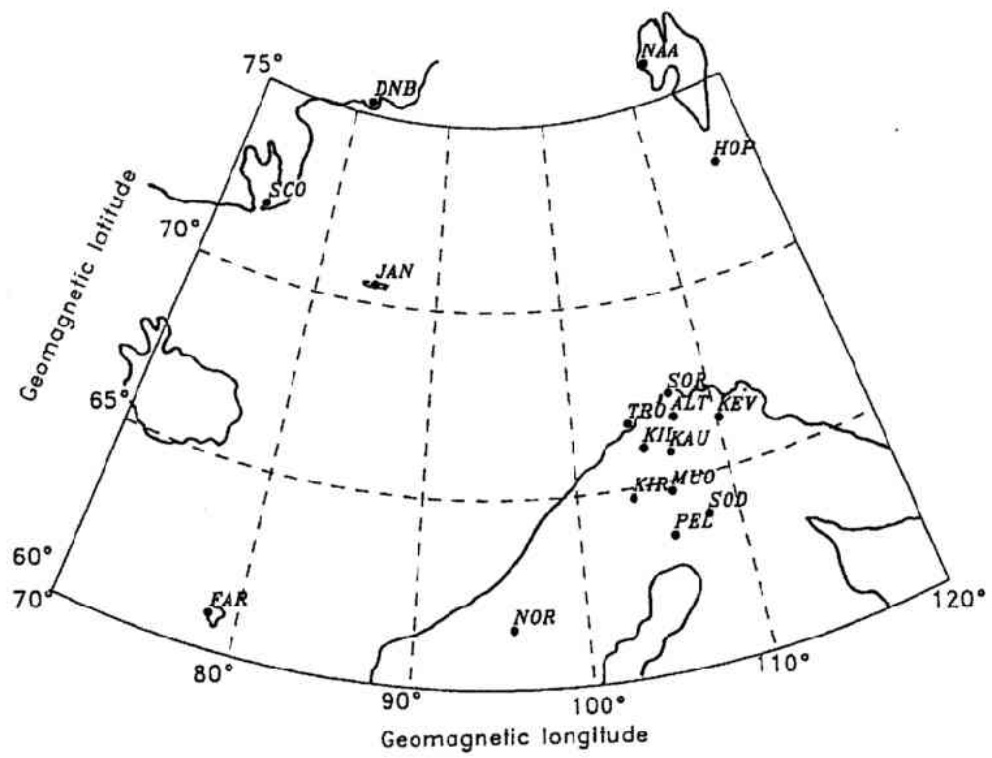

Fig. 3. Map of the magnetometer stations, whose data are used for the regional estimation, in geomagnetic coordinates. The EISCAT radar is located near Tromsø (TRO).

shown that the AMIE is used to derive the global pattern of ionospheric quantities whereas the KRM is employed for their regional estimation. Magnetograms from 81 stations in the northern hemisphere and those from 17 stations near northern Scandinavia (Fig. 3) are, respectively, utilized for the AMIE and for the regional estimation by the KRM. The grid size for the AMIE calculation is $1.7^{\circ}$ and $10^{\circ}$ in the geomagnetic latitude and longitude, respectively, while it is $1.0^{\circ}$ and $2.0^{\circ}$ for the regional estimation.

The current function is derived from ground magnetic records from the Scandinavian stations which are densely distributed. The boundary condition for solving a equation in terms of the current function is adopted from the global potential patterns. Equation (4) is solved for appropriate conductance distributions under a boundary condition to obtain the electric potential in the localized region. The boundary condition for (4) is given from the global AMIE calculation. Once the electric potential in the localized region is obtained, we can calculate the electric field and then the ionospheric current from (3). The divergence of the ionospheric current gives the field-aligned current such as:

$$
j_{\|}=\nabla \cdot \boldsymbol{J}
$$

where positive (negative) $j_{\|}$indicates the downward (upward) field-aligned current.

\subsection{Conductance distribution}

To solve (4) for the electric potential in a localized region, the distribution of the Hall and Pedersen conductances for that region is required. For this purpose, we make use of the data of the electron density measured by the EISCAT radar and of the precipitating electrons observed by the DMSP satellites.

The conductance distribution is determined in the following way: The conductances resulting from auroral precipitation can be estimated from the energy flux and average energy of the electrons obtained by DMSP by using an empirical formula of Robinson et al. (1987). We first assume that the conductances do not depend on longitude. The ionospheric parameters are then calculated from (3), (4) and (5) by using these first-approximation conductances. In the next step where we assume that the conductance enhancements are related to the density of upward field-aligned currents, the ionospheric conductances 
are modified in such a way as:

$$
\Sigma_{1}=\sqrt{\left(\Sigma_{0}\right)^{2}+\left(\alpha j_{\|}\right)^{2}} \quad\left(j_{\|}<0\right)
$$

where $\Sigma_{0}$ and $\Sigma_{1}$ are the first-approximation conductance and the modified conductance, respectively. The proportional coefficient $\alpha$ is determined by assuming that $\Sigma_{1}$ at the nearest grid point to the EISCAT radar is equal to the value observed by the radar: for the conductance calculation from the radar data, see Brekke and Hall (1988). By using the modified conductances which have the spatial dependence, (4) is solved again. We repeat these steps until the difference between the "old" and "new" potential values is reduced to $10 \%$.

\section{Application}

The method given in the previous section is applied to a substorm event which occurred near EISCAT at 2010 UT, April 9, 1990.

\subsection{A substorm event}

Three-component magnetic perturbations at the EISCAT magnetometer chain observatories for this substorm are shownin Fig. 4. The $H$ variations indicate that a westward electrojet activation started at 2010 UT along this meridian. Positive $D$ changes also started at the same time. About ten minutes after the
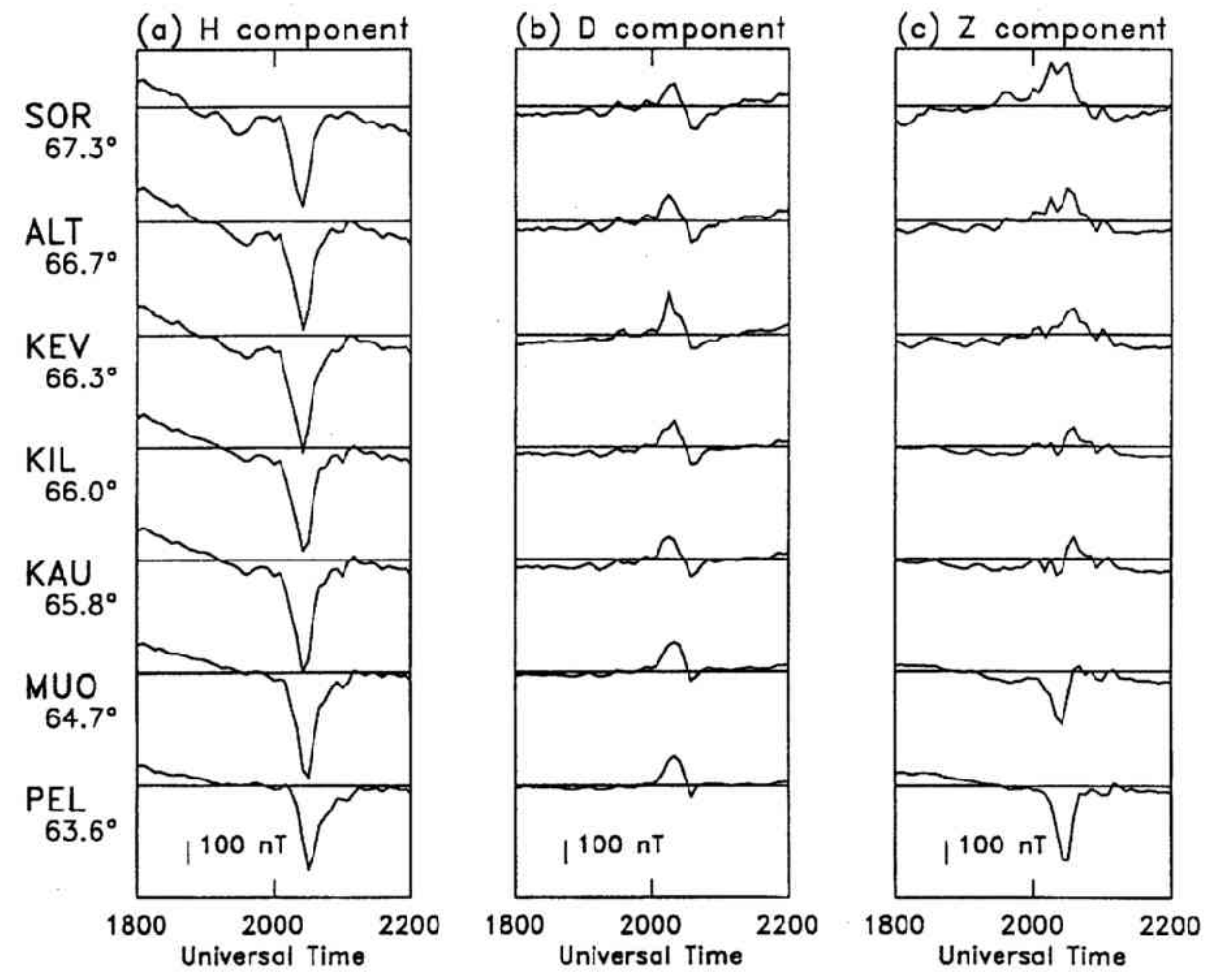

Fig. 4. Three-component magnetic perturbations observed at the EISCAT chain stations on April 9, 1990. The geomagnetic latitude of each station is indicated (cf. Fig. 3). A substorm started around 2010 UT, as seen in the enhanced negative $H$ component perturbations. 


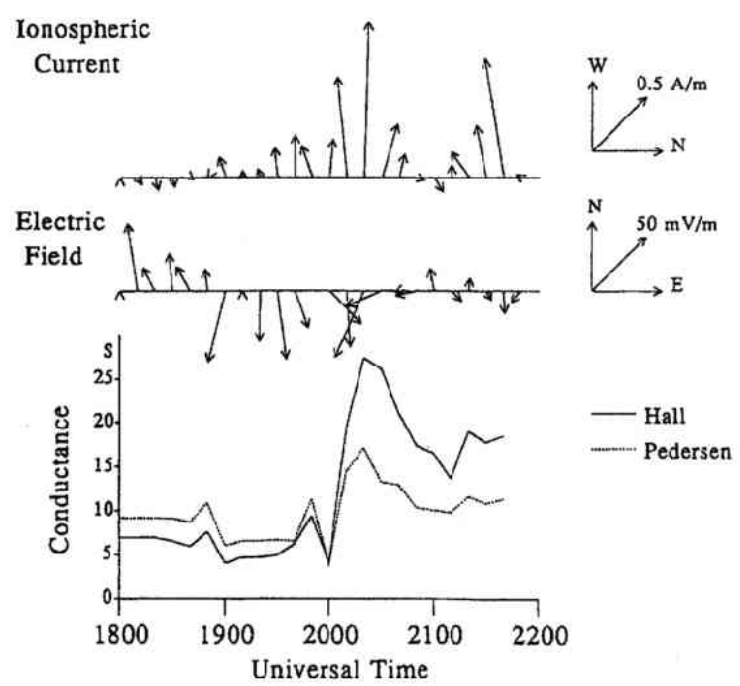

Fig. 5. Variations of the height-integrated ionospheric current, the electric field, and the Hall and Pedersen conductances obtained by the EISCAT CP-1 experiment on April 9, 1990. Note that upward vectors for the current and for the electric field indicate the westward and northward directions, respectively. Corresponding to the sudden enhancements in the conductances and the westward current around 2010 UT, the electric field changes its direction to westward.

positive changes, the $D$ component became negative at latitudes higher than $63^{\circ}$. A positive $Z$ excursion was recorded at Soroya and Alta, and a negative $Z$ perturbation was observed at Muonio and Pelo, implying that the center of the westward electrojet was located near $66^{\circ}$ during the expansion phase of the substorm. Such $D$ and $Z$ perturbations are typical near the westward traveling surge (Rostoker $e t$ al., 1980). The latitude of the electrojet center appears not to move considerably during this event.

During this interval, the EISCAT radar measured the electron density, electron and ion temperatures, and ion velocities with the CP-1 mode. These parameters are used to estimate current densities, conductivities and electric fields in the ionosphere. The electric field is calculated from the ion velocity in the $F$ region, which is approximately equal to the electron velocity determined by the $\boldsymbol{E} \times \boldsymbol{B}$ drift. Note that it takes about 10 minutes for the radar system to obtain a full set of ion velocities.

Figure 5 shows time variations of the height-integrated currents, the electric field and the Hall to Pedersen conductances for the interval under study. Before the substorm onset, the electric field of 50$60 \mathrm{mV} / \mathrm{m}$ was directed southward near the EISCAT site. Corresponding to the substorm expansion, the electric field changed its direction to southwestward and the intensity became smaller, to about $25 \mathrm{mV} /$ $\mathrm{m}$. Such a westward change often appears just after the substorm expansion onset (e.g., Robinson and Vondrak, 1990). The ratio of the Hall and Pedersen conductances during the event was 1.5-2.0, indicating that the average energy of precipitating electrons is from 3 to $6 \mathrm{keV}$.

The direct measurements of the energy spectra of precipitating electrons were made by the DMSP8 and -9 satellites which passed over northern Scandinavia around 2040 and 2055 UT, respectively. In fact, the observed precipitation patterns for 2040 UT and 2055 UT were very different, since the orbit of 2040 UT was about $400 \mathrm{~km}$ west of that of $2055 \mathrm{UT}$. Although the substorm had started to recover by $2055 \mathrm{UT}$, we have decided to make use of the energy spectra obtained at about 2055 UT in deriving the conductance distribution for the expansion phase for the following reasons: (1) The orbit at 2055 UT was much closer to the EISCAT site than that at 2040 UT. (2) The conductances derived from EISCAT and from DMSP9 were in good agreement. (3) As shown above, the location of the westward electrojet scemed not to move considerably throughout this event. As described in Subsection 2.2, the conductance distribution derived from the satellite data is used as the first-guess input to our scheme and then the distribution is optimized by using the EISCAT data. 


\subsection{Results of the regional estimation}

Figure 6 shows the electric potential distribution for 2020 UT, which is the maximum epoch of the substorm. This is derived by the AMIE technique, in which five-minute average data from 81 ground magnetometer stations are used. The overall potential pattern consists of two major cells at high latitudes. Associated with the enhanced substorm electrojet, the high potential cell intrudes into the premidnight sector, extending to 2100 MLT.

The electric potential in the localized region is solved for the boundary condition, which is adopted from potential values along a thick line in Fig. 6 . In order to examine quantitatively the dependence of the boundary condition and the conductance distributions on the solution, the calculation results for three quantities (the electric potential, current vectors, and field-aligned currents) are shown in Fig. 7 for the following four cases:

(a) The potential is set to be 0 on the boundary. Uniform conductances $\left(\Sigma_{\mathrm{H}}=8, \Sigma_{\mathrm{P}}=4\right)$ are employed.

(b) Along the boundary, the electric potential is given as $\psi / \Sigma_{\mathrm{H}}$ which is the solution of (4) for no conductance gradients over the region. The conductance distribution is the same as in (a).

(c) A more realistic boundary condition derived from the global AMIE calculation is used. The conductance distribution is the same as in (a).

(d) The realistic conductance distribution is employed, as described in Subsections 2.2 and 3.1. The boundary condition is given from the AMIE calculation.

Four rows (a), (b), (c) and (d) in Fig. 7 correspond to these four cases. It is clear that the boundary condition in Case (a) makes large distortions in the electric potential pattern over the distribution (b), which is the same as the current function, i.e., an input of the calculation. The electric potential patterns for (b) and (c) are similar, but the intensity of the electric field in (b) is $15 \%$ lower than that in (c) near the region where magnetometer stations are densely distributed. The difference in the ionospheric current

\section{ELECTRIC POTENTIAL}

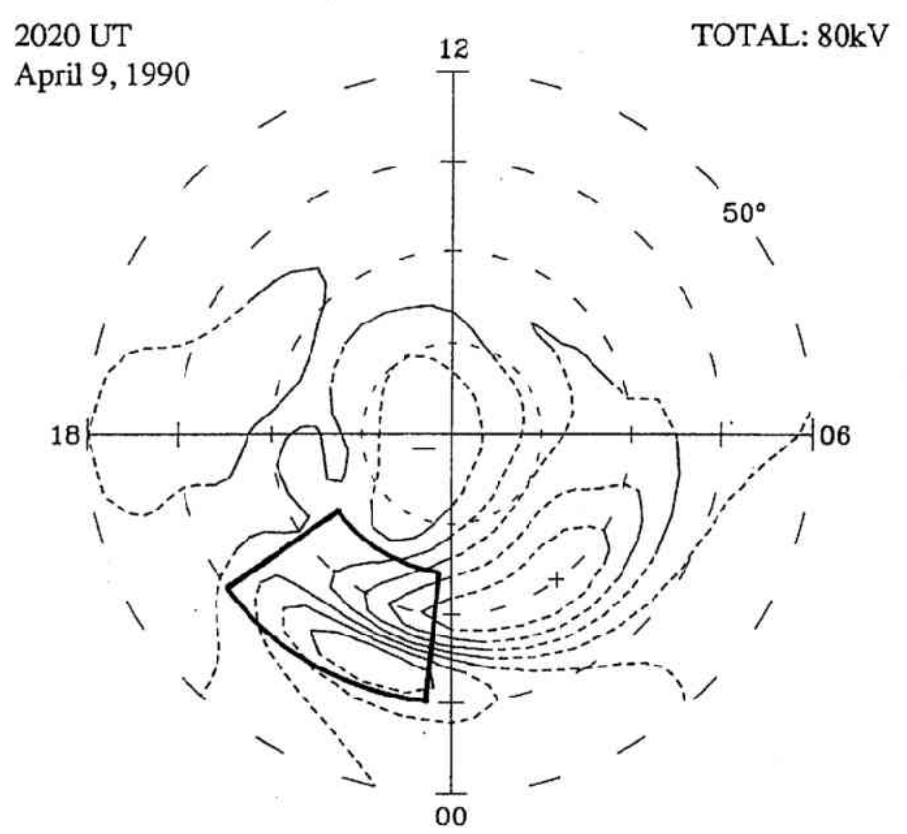

Fig. 6. Global electric potential pattern ( $10 \mathrm{kV}$ contour interval) over the polar region for $2020 \mathrm{UT}$ on April 9,1990 derived by the AMIE. A thick line indicates the boundary of the region in which electrodynamic parameters shown in Fig. 7 are estimated in detail. The contours are dashed where the estimated electric field uncertainty exceeds $50 \%$. 

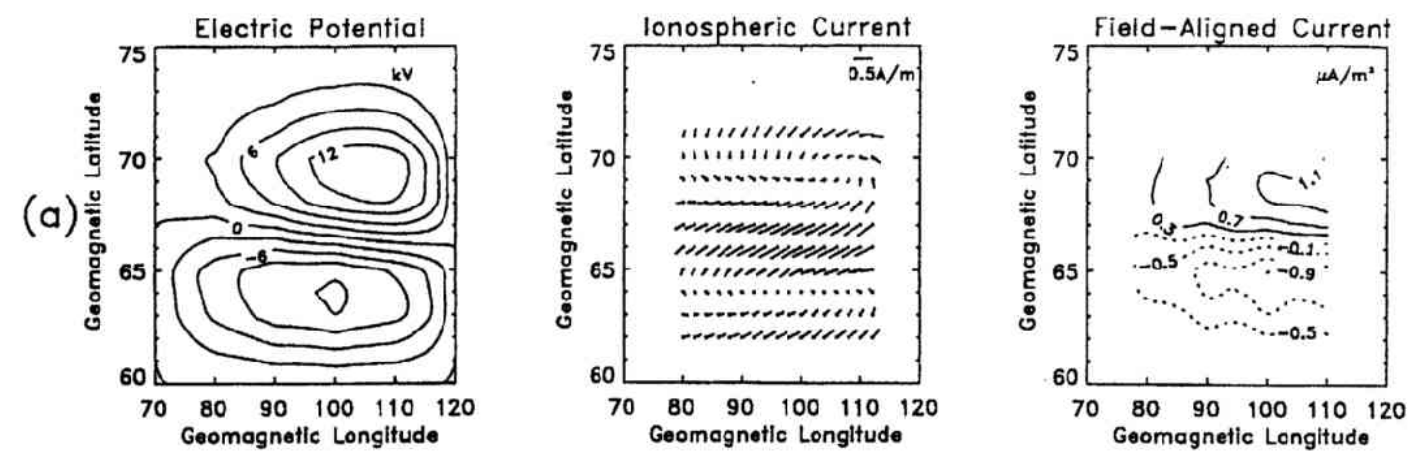

(b)
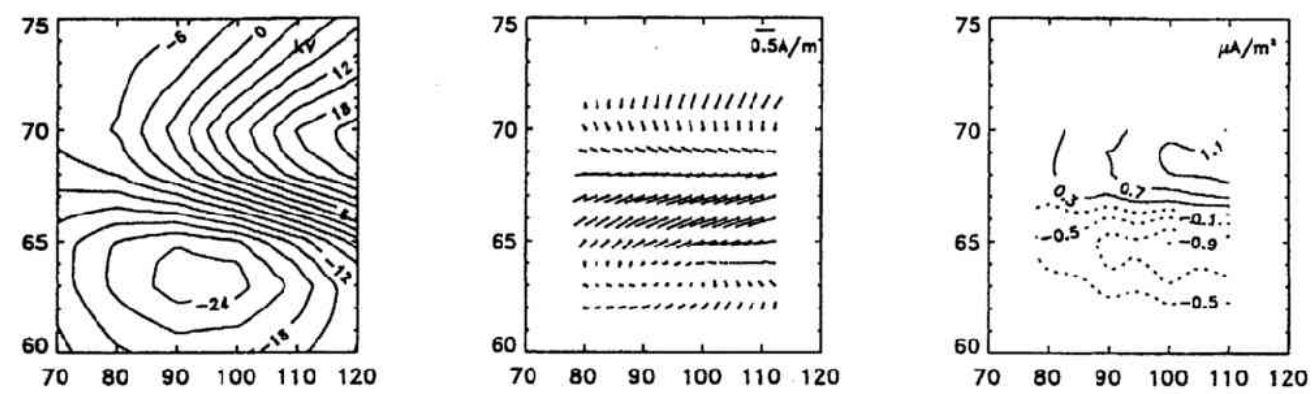

(c)
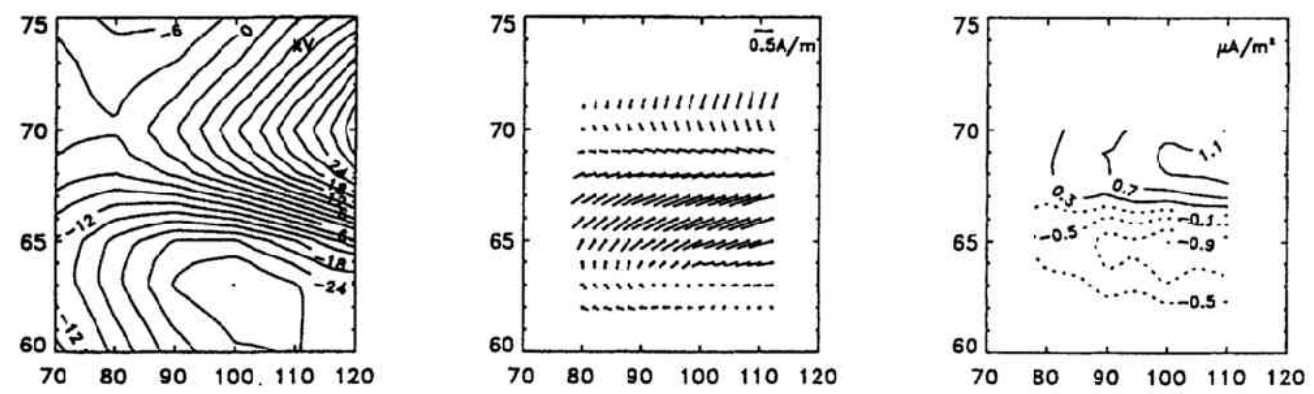

(d)
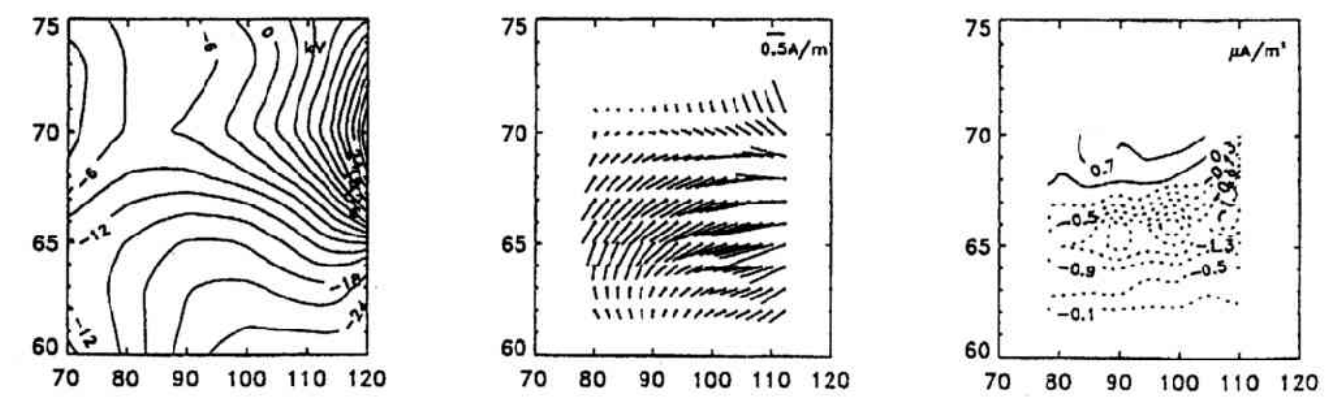

Fig. 7. Electric potential, current density vectors and field-aligned currents in the localized region for 2020 UT on April 9,1990 for four different cases. (a), (b), (c), and (d) correspond to the four cases of different boundary conditions and conductance inputs (see text for details). The ionospheric current and field-aligned current distributions are shown only for a region where the boundary condition effects are small. Contour intervals for the electric potential and for the field-aligned current are 3 $\mathrm{kV}$ and $0.4 \mu \mathrm{A} / \mathrm{m}^{2}$, respectively. The solid and dotted lines for the field-aligned current distribution indicate downward and upward currents, respectively. 


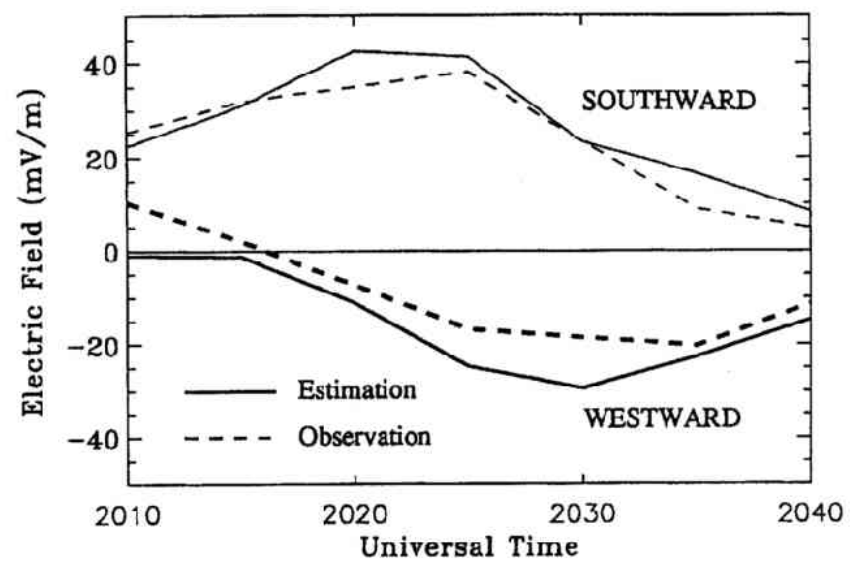

Fig. 8. Comparison between electric fields from the regional estimation and measured by the EISCAT radar. Their north-south and east-west components are plotted separately. The solid and dashed lines indicate the estimated and observed values, respectively. Note that the estimated values at the nearest point to EISCAT are plotted in every five minutes, while the observed values are plotted in every ten minutes.

intensity between (b) and (c) are comparable to that in the electric field. In contrast to patterns in the electric potential and the ionospheric current, the field-aligned current patterns in (a), (b) and (c) are almost identical, indicating that the field-aligned current distribution is not very sensitive to the boundary conditions.

From a comparison between the electric potential patterns for (c) and (d), it is seen that the calculated potential changes greatly depending on different conductances. The conductance for (d) is highly enhanced, especially in the region between $63^{\circ}$ and $68^{\circ}$ in geomagnetic latitude and between $90^{\circ}$ and $110^{\circ}$ in longitude. In this region, the difference between (c) and (d) in the electric field intensity is more than $50 \%$, whereas that in the current density is only about $25 \%$. The field-aligned current distribution also varies significantly since the field-aligned current for (d) includes contributions from conductance gradients: see Sato et al. (1995).

In Case (d), the westward component of the electric field becomes large within the enhanced conductance region. The current densities ranging from 0.7 to $1.2 \mathrm{~A} / \mathrm{m}$ in (d) are in good agreement with the observed values by EISCAT. Intense upward currents whose density is over $2.0 \mu \mathrm{A} / \mathrm{m}^{2}$ appear near $65^{\circ}$ where both absolute values and gradients of the conductances are relatively high.

In order to check the validity of the calculations, the estimated electric field for Case (d) is compared in Fig. 8 with what were actually observed by EISCAT. Similar comparisons have been made for Case (a), (b) and (c), finding larger differences between the observed and estimated values than those for Case (d). In the figure, the north-south and east-west components of the electric field at the grid point nearest to EISCAT are plotted for the comparison. Note that the five-minute average data are used in the regional calculation while the EISCAT measurements were carried out in a ten-minute resolution. It is evident that the estimated values of the electric field are in reasonable agreement with those obtained by the EISCAT measurements of the ion velocities. We feel, however, that this comparison is only for one point, so twodimensional measurements of the ion velocities by EISCAT CP- 2 and CP-3 mode operations are needed for a more critical comparison.

\section{Discussion}

Two magnetogram-inversion algorithms, i.e., AMIE and KRM, have been combined to estimate electrodynamic parameters in the ionosphere, making it possible to deduce distributions of global plasma 
convection and of localized structures of the parameters associated with substorms. In this proposed scheme, the EISCAT data play a major role in deriving the "absolute" values of the ionospheric conductances as well as in evaluating the accuracy of our calculations. In this section, we discuss briefly the capability, advantages and limitations of our new scheme.

\subsection{Boundary condition}

One of the advantages of our new scheme is that the boundary condition for the regional estimation is adopted from the global potential patterns so that both large-scale and small-scale structures of ionospheric processes can be discussed on a common ground. As shown in Subsection 3.2, the boundary condition for the regional estimation has an important influence on the results. This problem was discussed to some extent by Murison et al. (1985), who applied a magnetogram-inversion method to the Scandinavian Magnetometer Array. They showed that even when the boundary was set at over $500 \mathrm{~km}$ far from a localized region, the electric potential patterns in the region are affected strongly by the given boundary condition.

Figure 7 shows that not only the overall potential pattern but also the intensity of the electric field vary significantly, depending on different boundary conditions. In our scheme, the electric potential within a localized region as well as over the polar region are obtained in a consistent manner by combining the two inversion schemes. The electric field calculated with the boundary conditions determined by the AMIE is in good agreement with that observed by EISCAT.

\subsection{Improved conductance input}

Outputs of our calculation, particularly the electric potential, are dependent strongly on the choice of the ionospheric conductances. Therefore, accurate knowledge of the conductances is required in our scheme, although no direct measurements are presently able to obtain the instantaneous, two-dimensional distribution of the conductance. However, the recent availability of radar and satellite data of ionospheric parameters provides us with an opportunity to make an optimized estimate of the ionospheric conductance. In Section 3, we have used data from the EISCAT radar and the DMSP satellite to determine the conductance distribution. It is reasonable that changes in the precipitating particle flux along satellite orbits are regarded as the spatial distribution, since the polar-orbiting satellites pass over northern Scandinavia within only four minutes. This time scale is comparable to the time interval over which magnetometer data are averaged in the present study. We feel, however, that uncertainties about the longitudinal distribution of precipitating particles still remain. Moreover, the probability of satellite's passages over Scandinavia at the right time is quite low, making it difficult to derive a complete picture of electrodynamic patterns for the different substorm phases.

For these reasons, the EISCAT radar is crucially important in providing time variations of the ionospheric conductances at a fixed location. Although we have made use of data from the EISCAT's CP1 observations in this paper, data from the CP-2 mode of measurements are even more useful since they provide horizontal gradients of the electron density in both the longitudinal and latitudinal directions.

\subsection{Global and localized distributions}

We have demonstrated in Subsection 3.2 how our method can be applied to a substorm event, resulting in the instantaneous distribution of the substorm westward electrojet in a localized region as well as distored two-cell convection patterns. The regional estimation has been successful in deducing the small-scale patterns of the ionospheric parameters. Even though our ability to determine accurately the current patterns depends on how well the magnetometer stations are distributed, it may still be possible for our scheme to derive the electrodynamic properties with the spatial accuracy of about $100 \mathrm{~km}$, which is comparable to the ionospheric height and to the size of an active aurora associated with substorm onset as observed by the satellites (e.g., Murphree et al., 1991).

Figure 7(d) shows a localized upward current which is related to auroral electron precipitation associated with the substorm expansion. By comparing the localized distribution with the global pattern 
shown in Fig. 6, it is noted that the substorm westward electrojet is located in a somewhat equatorward portion of the general two-cell pattern, distorting the overall potential pattern in the premidnight sector. This pattern is similar to that presented by Kamide et al. (1994), who have studied the dependence of the convection pattern on the different phases of substorms. They have shown that a pair of potential cells for enhanced global convection and another pair of cells for substorm expansion in the midnight sector can coexist during the substorm expansion phase.

A number of subjects on the substorm onset processes are still unsolved, such as the location of substorm initiation relative to the preexisting large-scale convection and the role of the electric field in the processes. For example, Robinson and Vondark (1990) showed that the auroral surge is formed within a region of the northward electric field, i.e., south of the convection reversal regions, and that the northward component is reduced within the surge. On the contrary, Lyons et al: (1990) argued that the auroral brightening occurs within the region several degrees poleward of the electric field reversal. Both of them did rely on radar measurements as well as on ground-based and satellite-based observations of auroral images which provide simultaneous data of the electric field and particle precipitations, but it was rather difficult to differentiate spatial and temporal changes in individual cases of radar observations. Instantaneous global distributions of the electric field are required to discuss their problems. In this connection, our scheme is very useful to examine the relationship between the plasma convection before the substorm expansion onset and localized upward currents associated with the auroral breakup.

In terms of energy transfer processes from the solar wind to the ionosphere through the magnetosphere, global plasma convection is regarded as a manifestation of the "directly driven" process, and substorm initiation enhancing the westward electrojet in the midnight sector is a consequence of the "unloading" process (e.g., Rostoker et al., 1987). Therefore, by deducing global and localized potential patterns for the same time, our scheme is expected to lead us to an understanding of how those two processes couple with each other throughout substorm activity.

We would like to thank A. D. Richmond and A. Brekke for their helpful discussions throughout the project reported in this paper. The work of M.S. was supported by the Research Fellowships program of the Japan Society for the Promotion of Science for Young Scientists. This work was supported in part by the Scandinavia-Japan Sasakawa Foundation.

\section{REFERENCES}

Baumjohann, W., R. L. Pellinen, H. J. Opgenoorth, and E. Nielsen, Joint two-dimensional observations of ground magnetic and ionospheric electric fields associated with auroral zone currents: Current systems associated with local auroral breakups, Planet. Space Sci., 29, 431-447, 1981.

Brekke, A. and C. Hall, Auroral ionospheric quiet summer time conductances, Ann. Geophys., 6, 361-376, 1988.

Kamide, Y. and W. Baumjohann, Magnetosphere-Ionosphere Coupling, Springer-Verlag, Heidelberg, 1993.

Kamide, Y., A. D. Richmond, B. A. Emery, C. F. Hutchins, B.-H. Ahn, O. de la Beajardiere, J. C. Foster, R. A. Heelis, H. W. Kroehl, and F. J. Rich, Ground-based studies of ionospheric convection associated with substorm expansion, J. Geophys. Res., 99, 19451-19466, 1994.

Kamide, Y., A. D. Richmond, and S. Matsushita, Estimation of ionospheric electric fields, ionospheric currents and field-aligned currents from ground magnetic records, J. Geophys. Res., 86, 801-813, 1981.

Lyons, L. R., O. de la Beajardiere, G. Rostoker, J. S. Murphree, and E. Friis-Christensen, Analysis of substorm expansion and surge development, J. Geophys: Res., 95, 10575-10589, 1990.

Murison, M., A. D. Richmond, S. Matsushita, and W. Baumjohann, Estimation of ionospheric electric fields and currents from a regional magnetometer array, J. Geophys. Res., 90, 3525-3530, 1985.

Murphree, J. S., R. D. Elphinstone, L. L. Cogger, and D. Hearn, Viking optical substorm signatures, in Magnetospheric Substorms, edited by J. R. Kan, T. A. Potemra, S. Kokubun, and T. Iijima, 241 pp., Geophysical Monograph 64, American Geophysical Union, 1991.

Richmond, A. D. and Y. Kamide, Mapping electrodynamics features of the high-latitude ionosphere from localized observations: Technique, J. Geophys. Res., 93, 5741-5759, 1988.

Robinson, R. M. and R. R. Vondrak, Electrodynamic properties of auroral surges, J. Geophys. Res., 95, 7819-7832, 1990.

Robinson, R. M., R. R. Vondrak, K. Miller, T. Dabbs, and D. Hardy, On calculating ionospheric conductances from the flux and 
energy of precipitating electrons, J. Geophys. Res., 92, 2565-2569, 1987.

Rostoker, G., S.-I. Akasofu, J. Foster, R. A. Greenwald, Y. Kamide, K. Kawasaki, A. T. Lui, R. L. McPherron, and C. T. Russell, Magnetospheric substorms-Definition and signatures, J. Geophys. Res., 85, 1663-1668, 1980.

Rostoker, G., S.-I. Akasofu, W. Baumjohann, Y. Kamide, and R. L. McPherron, The roles of direct input of energy from the solar wind and unloading of stored magnetotail energy in driving magnetospheric substorms, Space Sci. Rev., 46, $93,1987$.

Sato, M., Y. Kamide, A. D. Richmond, A. Brekke, and S. Nozawa, Regional-estimation of electric fields and currents in the polar ionosphere, Geophys. Res. Lett., 22, 283-286, 1995.

Untiedt, J. and W. Baumjohann, Studies of polar current systems using the IMS Scandinavian Magnetometer Array, Space Sci. Rev., 63, 245-390, 1993. 